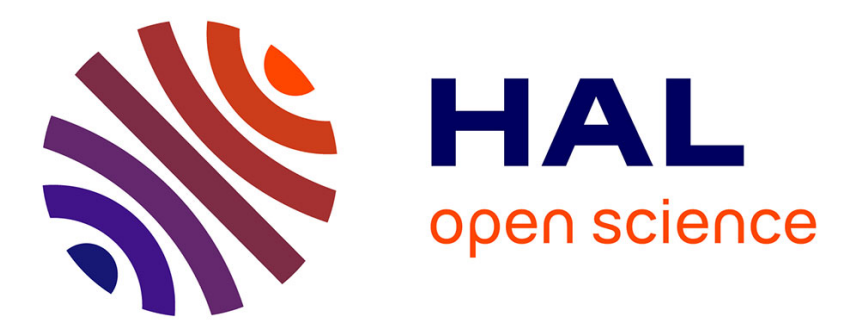

\title{
Stability Analysis of a Nonlinear System with Infinite Distributed Delays Describing Cell Dynamics
}

Walid Djema, Frédéric Mazenc, Catherine Bonnet, Jean Clairambault, Emilia Fridman

\section{- To cite this version:}

Walid Djema, Frédéric Mazenc, Catherine Bonnet, Jean Clairambault, Emilia Fridman. Stability Analysis of a Nonlinear System with Infinite Distributed Delays Describing Cell Dynamics. ACC 2018 - American Control Conference, Jun 2018, Milwaukee, United States. 10.23919/acc.2018.8430869 . hal-01849010

\author{
HAL Id: hal-01849010 \\ https://hal.inria.fr/hal-01849010
}

Submitted on 25 Jul 2018

HAL is a multi-disciplinary open access archive for the deposit and dissemination of scientific research documents, whether they are published or not. The documents may come from teaching and research institutions in France or abroad, or from public or private research centers.
L'archive ouverte pluridisciplinaire HAL, est destinée au dépôt et à la diffusion de documents scientifiques de niveau recherche, publiés ou non, émanant des établissements d'enseignement et de recherche français ou étrangers, des laboratoires publics ou privés. 


\title{
Stability Analysis of a Nonlinear System with Infinite Distributed Delays Describing Cell Dynamics
}

\author{
W. Djema \\ F. Mazenc \\ C. Bonnet \\ J. Clairambault \\ E. Fridman
}

\begin{abstract}
We want to reconcile some earlier modeling ways of the cell cycle in one common framework. Accordingly, we consider a model that contains a compartment where cells may be quiescent for an unlimited time, along with a proliferating phase in which most of the cells may divide, or die, while few of them may be arrested during their cycle for unlimited time. In fact, the cell-cycle arrest may occur for many reasons (DNA damages detected at some checkpoints, insufficient resources for cell grow, drug infusions). We actually extend some early models involving finite distributed delays (taken from [2], [1]) to the case of infinite distributed delays and time-varying parameters. Our main result relies on the construction of a novel Lyapunov-Krasovskii functional, suitable for the analysis of the origin of the system involving infinite distributed delays and time-varying parameters.
\end{abstract}

Key Words: Infinite Distributed Delay, LyapunovKrasovskii Functionals (LKFs), Exponential Stability.

\section{INTRODUCTION}

Cells that engage in the division process (cell cycle, [16]) usually undergo a series of active transformations and a mechanism of nuclear division (mitosis), that ends with the division of each mother cell into two daughter cells (cytokinesis, [22]). The cell cycle leads to the growth of tissues in all multicellular organisms. A small category of adult cells is known as stem cells (SCs): these are undifferentiated cells characterized by their extensive ability to self-renew and their multipotency, which is the ability to differentiate into more specialized cells [27]. When cells become more and more mature, they gradually lose their self-renewal feature. Even in fast-renewing tissues, most of the cells are in fact in a non-proliferating state known as the resting or quiescent phase, $G_{0}[22]$. A resting cell prepares its entrance into the cycle, which is formed by four consecutive discrete subphases $G_{1}, S$, $G_{2}$ and $M$ (see [8], Chap. 17), where the $M$-phase stands for mitosis, or nuclear division. Several checkpoints are used by the body to control cells that undergo division. These checkpoints gather a set of regulatory proteins that

Walid Djema* corresponding author, walid.djema@inria.fr. Walid Djema, Frédéric Mazenc and Catherine Bonnet are with Inria, University Paris-Saclay, L2S (CNRS), CentraleSupélec, Gifsur-Yvette, France. Jean Clairambault is with Inria, University of Sorbonne, UPMC, Laboratory Jacques-Louis Lions, Paris, France. Emilia Fridman is with the Department of Electrical Engineering and Systems at the School of Electrical Engineering, Tel-Aviv, Israel. walid.djema@inria.fr, frederic.mazenc@inria.fr catherine.bonnet@inria.fr, jean.clairambault@inria.fr emilia@eng.tau.ac.il monitor the progression of cells through different stages in the cycle. If some requirements are not fulfilled at specific moments, the regulatory proteins network takes over the issue by preventing the cell progression through the cycle. Consequently, abnormal cells (e.g. the ones with damaged DNA) cannot move forward in the division process, causing cell arrest. These cells are then obliged to undergo a process of DNA repair, or they are doomed to die by apoptosis.

There are in fact three main checkpoints: $G_{1} / S$, $G_{2} / M$, and the metaphase checkpoints. In the $G_{1} / S$ checkpoint, the cell ensures that it has enough materials to replicate its DNA. Thus, unhealthy or malnourished cells are stuck at this point. Similarly, in the $G_{2} / M$ checkpoint, the cell ensures that it has enough cytoplasm and phospholipids for two (daughter) cells, otherwise, it is prevented from performing mitosis. Lastly, at the metaphase checkpoint, the division is stopped if chromosomes are not all well-aligned at the spindle equator before anaphase. The length of the cycle is approximately 24 hours for fast-dividing mammalian cells. However, even when cells are not arrested, this duration may vary from one type of tissue to another, and even from one cell to another within the same tissue. Some authors focused on the length of $G_{1}$ and its possible applications ([19], [25]); e.g., the manipulation of the $G_{1}$ length in neural SCs is discussed in [25], including also its impacts on the differentiation of neural precursors. We refer to Fig. 1 in [19] that gives the lengths of the cell cycle (in particular the length of $G_{1}$ ) of some important categories of cells, including blood lineages, gut lineage and neural lineage. Note that, in the classification given in [19], the cycle length of the common myeloid progenitors (CMP) -in the hematopoietic system- appears as unknown or undetermined. We also emphasize that cancer dormancy is sometimes explained through cellular dormancy, i.e. $G_{0}$ and $G_{1}$-arrest [3], which might be also associated to the cell arresting power of some chemotherapies [24].

Our objective is to extend some modeling aspects in earlier models describing population cell dynamics. For that purpose, in this paper, we simply slightly modify the model presented in [2], [1], where all the cells have been assumed to divide or die before a finite-time during their cycle. So, here we consider that a minority of cells can be arrested for an infinite time within the division-cycle. Then, we generalize the stability analysis of the trivial steady state of the resulting system, from a finite to an infinite proliferating phase. 


\section{TRENDS AND MODELS OF THE CELL CYCLE}

We pointed out that the cell-cycle length can be different within the total population of cells, and that few cells may be arrested for an indefinite duration. In fact, some models are already taken into account a proliferative phase of infinite duration, while other models assume that all the cells are obliged to divide before a maximum finite age (see Fig. 1). Basically, the following questions may arise in this case: If some models take into account the cell arrest, while other models consider that all the cells must divide or die before a finite maximum age, can we expect equivalent representations in both frameworks? More precisely, does the minority of arrested cells cause a change in the asymptotic behavior of the model that has been assumed to have a finite proliferating phase?

Let us begin here with a brief review of some existing models of the cell cycle. Firstly, it is worth mentioning that some models in the literature were introduced in order to investigate the behavior of cells, undergoing proliferation, at their specific checkpoints, i.e. the interest was on the transitions from a given sub-phase of the cell cycle to the next one. Accordingly, some models consider some explicitly separated sub-phases of the cell cycle (see, e.g., [5], [4]). The latter perspective is in fact beyond the scope of this work. Indeed, here we consider a model that represents the transition between the resting phase $G_{0}$ (which is not explicitly modeled in [5]) and the proliferating compartment (i.e. the sum of $G_{1}, S, G_{2}$ and $\left.M\right)$, together with their respective lengths. We recall that cell population models with separated quiescent and proliferating compartments date back to some pioneers works such as Burns \& Tannock [7] and Mackey [20], which have been more recently improved by many authors, including Adimy et al. [2], [1], Özbay et al. [23], Djema et al. [13].

In Fig. 1, we point out some differences between two main modeling approaches: on the one hand, we observe the configurations (A) and (B) where the $G_{0^{-}}$ state is taking into account, and on the other hand, the configuration (C) where $G_{0}$ is assimilated to the phase $G_{1}$. These two trends may be - in some sense - reconciled by adopting the representation (D) (see the arguments given in Fig. 1). More precisely, the configuration (D) improves the one illustrated in $(\mathbf{C})$ by clearly separating the $G_{0}$ compartment from the cell cycle. In addition, contrary to (A) and (B), the representation (D) extends the representation of the cell cycle by considering it of unlimited length. Thus, the role of the growth phase $G_{1}$ and the gap phase $G_{2}$ (which are of variable duration in healthy and unhealthy cases, and which may halt some dividing cells) is taken into account. The configuration in (D)-Fig. 1 is the one that we want to study. Our first objective is to extend the LKF constructions we provided in [10] to the case of models involving infinite distributed delays, modeling the infinite proliferating phase.

In order to better understand the cell dynamics in healthy or unhealthy situations, mathematical modeling and analyses are generally required. In particular, since the aim of anti-cancer therapies is the eradication of all malignant cells, the focus is put on investigating the stability properties of the zero solution of the model (here it is about the model in (D)-Fig. 1). Indeed, the convergence of the model trajectories to zero means total-cells extinction, which reflects to desired fate for unhealthy and cancer cells. It is well known that theory of Lyapunov functions offers efficient tools to study nonlinear systems ([17], [21]), possibly involving delays ([15]). However, the drawback of this approach is that no systematic method exists to find a suitable Lyapunov function for a given nonlinear system (see [18], [21], [17] and [26]). At this juncture, we mention that the version of the biological model of interest, involving finite distributed delays (introduced and analyzed in [2], [1]), has been already investigated through the construction of strict Lyapunov-Krasvoskii functionals (LKFs) in [10] (for its two steady states: the trivial and the strictly positive ones). However, extending the LKF construction given in [10], to investigate the stability properties of the origin of the model involving infinite distributed delays, is not a trivial task.

\section{A NONLINEAR CELL POPULATION MODEL INVOLVING INFINITE DISTRIBUTED DELAYS}

The model of interest is the one illustrated in Fig. 2, in which we have $n$ distinguishable maturity levels $\left(i \in I_{n}=\right.$ $\{1, \ldots, n\}$, where $i=1$ is the SC-compartment). Some modeling aspects are simplified in this work since they deserve a separated study. More precisely, cell plasticity features are neglected in this paper (refer to [12]).

We study the following nonlinear system with infinite distributed delays:

$$
\begin{gathered}
\dot{x}_{i}(t)=-d_{r i}(t) x_{i}(t)-\beta_{i}\left(x_{i}(t)\right) x_{i}(t) \\
+2 \lambda_{i}(t) \int_{0}^{+\infty} g_{i}(t, a) \beta_{i}\left(x_{i}(t-a)\right) x_{i}(t-a) d a \\
+2 \sigma_{i-1}(t) \int_{0}^{+\infty} g_{i-1}(t, a) \beta_{i-1}\left(x_{i-1}(t-a)\right) \\
\times x_{i-1}(t-a) d a,
\end{gathered}
$$

where $f_{i}(a)=h_{i}(a) e^{-\int_{0}^{a} h_{i}(m) d m}$,

$$
g_{i}(t, a)=f_{i}(a) e^{-\int_{0}^{a} d_{p i}(m+t-a) d m},
$$

and $h_{i}$ is the cell-division rate. In [2], $h_{i}$ fulfilled the condition $^{1} \int_{0}^{\tau_{i}} h_{i}(a) d a=+\infty$, and it has been considered as a continuous non-decreasing function satisfying $\lim _{a \rightarrow \tau_{i}} h_{i}(a)=+\infty$. Now, in our work, we consider that the (continuous) cumulative distribution function $h_{i}$ is defined over an infinite support $[0,+\infty)$, is nondecreasing and it satisfies $\lim _{a \rightarrow+\infty} h_{i}(a)=1$. Indeed, we consider

\footnotetext{
${ }^{1}$ This assumption describes the fact that all the proliferating cells which do not die by apoptosis during the cell cycle, are obliged to divide before they reach the maximal age $\tau_{i}$.
} 


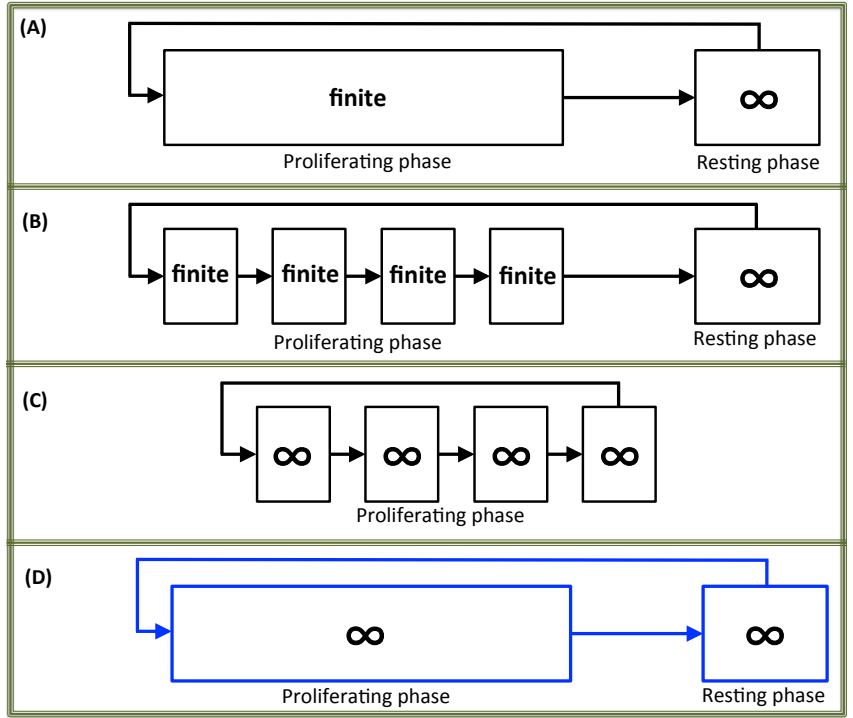

Fig. 1. Cartoon approximate representation of some cell cycle models from the literature. Configuration $(\mathbf{A})$ is the one in which cells may be quiescent during their entire life, or they can enter to a proliferating stage of finite length (as in [2], [1], [23], [10]). In (B), the cell cycle is splited into four sub-phases $G_{1}, S, G_{2}$ and $M$, each one has a finite duration (as in [4]), i.e. in (B) the length of the proliferating phase is finite since all the sub-phases were assumed to be finite. Next, the situation in (C) (as in [5]) is completeley different, since in this approach the cell cycle is described as an infinite support without an explicit resting phase $G_{0}$ (that can be associated with $G_{1}$ ). Thus, in (C), the lengths of the sub-phases $G_{1}, S, G_{2}$ and $M$ are infinite. Finally, we notice that (D) represents the case in which we consider that, at least, $G_{1}$ or $G_{2}$ may be of infinite lengths in the cell cycle, which is compacted in a single infinite phase, along with a separated infinite resting compartment $G_{0}$. In summary, one notices that the configuration (D) separates quiescence from proliferation (as in (A)-(B)), while proliferation is of infinite length (as in (C)). Some slightly different models are considered for instance in [6], where a molecular structured population (involving age-and-cyclin structured-PDEs) have been studied, considering that the length of the cell cycle is infinite.

that when the age $a$ increases, the cells which do not die by apoptosis have an increasing probability to divide, and in fact the probability of division goes to 1 when $a$ goes to infinity. The latter description captures the fact that a majority of cells may divide (if they do not die) and few of them may be arrested within the cycle. Next, we assume that $\lambda_{i}(t) \in\left[\underline{\lambda}_{i}, \bar{\lambda}_{i}\right] \subset(0,1), \sigma_{i}(t) \in\left[\underline{\sigma}_{i}, \bar{\sigma}_{i}\right] \subset(0,1)$, and $\lambda_{i}(t)=1-\sigma_{i}(t)$. Moreover, we consider that for all $t \geq 0$, the death rates satisfy: $d_{r i}(t) \in\left[\underline{d}_{r i}, \bar{d}_{r i}\right] \subset(0, \infty)$, and $d_{p i}(t) \in\left[\underline{d}_{p i}, \bar{d}_{p i}\right] \subset(0, \infty)$. A direct consequence is that

$$
e^{-a \bar{d}_{p i}} \leq e^{-\int_{0}^{a} d_{p i}(m+t-a) d m} \leq e^{-a \underline{d}_{p i}} .
$$

Then, for all $t \geq 0$, and for all $a \in[0,+\infty)$,

$$
f_{i}(a) e^{-a \bar{d}_{p i}} \leq g_{i}(t, a) \leq f_{i}(a) e^{-a \underline{d}_{p i}} .
$$

The mitosis function $f_{i}$ satisfies $\int_{0}^{+\infty} f_{i}(a) d a=1$. This basically means that cells which do not die by apoptosis are intended to divide, but in infinite time.

For all $i \in I_{n}$ and $t \geq 0, d_{r i}(t)$ is the death rate of the resting cells, while $d_{p i}(t)$ is the death rate of proliferating

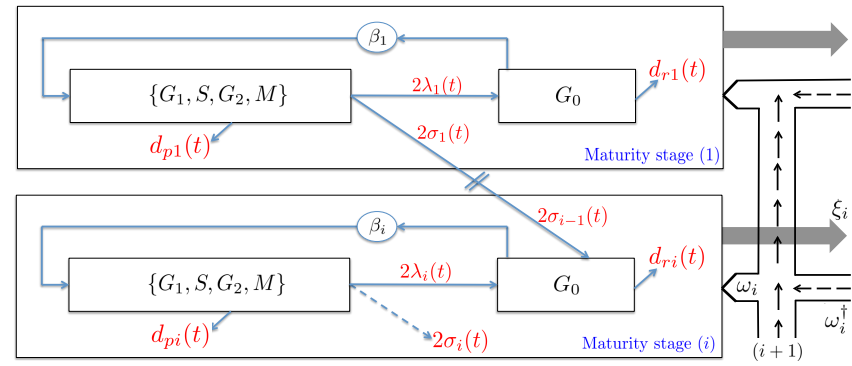

Fig. 2. A cartoon representation of the discrete maturity model of interest. Cells of the $i$-th maturity-generation, where $i \in I_{n}=$ $\{1, \ldots, n\}, n>1$, are in a resting phase $G_{0}$, or in proliferation (celldivision cycle) $\left\{G_{1}, S, G_{2}, M\right\}$ of infinite support (i.e. few cells may be arrested in the cell cycle for unlimited time). In the general case, we can consider that all the involved biological parameters (rate of differentiation $\sigma_{i} \in(0,1)$, rate of self-renew $\lambda_{i}=1-\sigma_{i}$, apoptosis rate $d_{p i}$, death rate of resting cells $d_{r i}$ ) are time-varying. On the right, the flux due to the cell-plasticity functions $\left(\xi_{i}\right.$ and $\omega_{i}$ for all $\left.i \in I_{n}\right)$ is represented. However, in the current paper, all these plasticity feature are ignored here: these issues are in fact discussed in a separated study in [12]. Thus, the model on which we focus in the present paper extends the one in [2] to the case of time-varying parameters and to the situation in which a minority of cells may be arrested for an infinite time in the cell cycle.

cells. The reintroduction function $\beta_{i}$ is decreasing and $\lim _{\ell \rightarrow \infty} \beta_{i}(\ell)=0$. The function $\xi_{i}$ describes the cell loss by cell plasticity mechanisms, that we assume in this work to be negligible when compared to $d_{r i}$. Next, $\sigma_{i}(t)$ represents the time-varying rate of differentiation and, consequently, $\lambda_{i}(t)=1-\sigma_{i}(t)$ is the rate of self-renewal of the $i$-th cell generation. Similarly to $\xi_{i}$, we consider that $\omega_{i}$, which quantifies the input from cell-plasticity features, is negligible in this work. We invite the interested reader to refer to [12] for more detail on plasticity functions related to cancer. Finally we notice that in our work, apart from the aforementioned extension to the case of unlimited cell-cycle length, we are also considering that all the biological parameters (differentiation, selfrenewing and apoptosis rates), involved in our model, are time-varying, which is not the case of the similar earlier models ([20], [2], [23]).

Remark 1: The system (1) is positive, i.e. for positive initial conditions the trajectories are positive for all $t \geq 0$ (see for instance a similar proof in the case of finite distributed delays in Proposition 1, [11]). Throughout the work, we consider only positive solutions of (1). The positivity of system (1) makes it possible to use some unusual non-quadratic Lyapunov functionals, which can be approximated at the origin by linear functionals [10].

\section{Stability ANAlysis of The 0-EQUilibrium}

In this section, we analyze the stability properties of the origin of the model (1), since the aim of anticancer therapy is the eradication of unhealthy cells. More precisely, we prove in this section the following result:

Theorem 1: Let $\bar{C}_{i}=\int_{0}^{+\infty} f_{i}(\ell) e^{-d_{p i} \ell} d \ell$. The system (1) admits the 0-equilibrium as a globally exponentially 
stable equilibrium point if the inequalities:

$$
\underline{d}_{r i}-\left(2 \bar{\lambda}_{i} \bar{C}_{i}-1\right) \beta_{i}(0)>0,
$$

are satisfied for all $i \in I_{n}=\{1, \ldots, n\}$. If

$$
\underline{d}_{r 1}-\left(2 \bar{\lambda}_{1} \bar{C}_{1}-1\right) \beta_{1}(0)<0,
$$

then no positive solution converges to 0-equilibrium.

Corollary 1: (i) Assume that the conditions

$$
2 \bar{\lambda}_{i} \bar{C}_{i}-1<0,
$$

are fulfilled for all $i \in I_{n}=\{1, \ldots, n\}$, then the trivial steady state is globally exponentially stable.

(ii) Assume that the conditions

$$
2 \bar{\lambda}_{i}-1<0,
$$

are fulfilled for all $i \in I_{n}=\{1, \ldots, n\}$, then the trivial steady state is globally exponentially stable.

Remark 2: It is clear that if the inequalities (8) are satisfied, then the conditions (7) are also satisfied (since $\bar{C}_{i}<1$, as $\underline{d}_{p i}>0$ ). Similarly, we see that if the conditions (7) hold true then those in (5) are also verified, since $\underline{d}_{r i}>0$. In fact, the conditions (5) are the direct generalization of the conditions $2 L_{i} C_{i}-1>0$ in [2] (see also [23] and [10]) to the case of infinite distributed delays and time-varying parameters. Biologically, one readily check that the conditions (8) are extreme conditions that ensures exponential eradication of unhealthy cells even if apoptosis is stalled to zero (i.e. $d_{p i}=0$ and $\bar{C}_{i}=1$ ).

Remark 3: The condition (5) is a direct generalization of the well-known necessary and sufficient stability condition for the 0-equilibrium of the class of systems with finite distributed delays and constant parameters (introduced in [2]). More precisely, the same kind of stability condition has been provided in [2] for local asymptotic stability of the origin, and in [10] for global exponential stability of the origin, of the model with finite distributed delays. However, proving an equivalent result in the case of infinite delays requires a novel Lyapunov-Krasovskii functional, slightly different from the one already used in [10].

Proof: The proofs of Theorem 1 and Corollary 1 are not provided in this brief version (refer to [9]).

\section{REFERENCES}

[1] Adimy, M., Crauste, F. El Abdllaoui, A., 2010. Boundedness and Lyapunov function for a nonlinear system of hematopoietic stem cell dynamics. Comptes Rendus Mathematique, 348(7-8), pp.373-377.

[2] Adimy, M., Crauste, F. and El Abdllaoui, A., 2008. Discretematurity structured model of cell differentiation with applications to acute myelogenous leukemia. Journal of Biological Systems, 16(03), pp.395-424.

[3] Aguirre-Ghiso, J.A., 2007. Models, mechanisms and clinical evidence for cancer dormancy. Nature reviews. Cancer, 7(11), p.834.

[4] Avila, J.L., Bonnet, C., Clairambault, J., Özbay, H., Niculescu, S.I., Merhi, F., Tang, R. and Marie, J.P., 2012. A new model of cell dynamics in Acute Myeloid Leukemia involving distributed delays. IFAC Proceedings Volumes, 45(14), p.55.
[5] Billy, F., Clairambault, J., Delaunay, F., Feillet, C. and Robert, N., 2012. Age-structured cell population model to study the influence of growth factors on cell cycle dynamics. Mathematical Biosciences and Engineering.

[6] Brikci, F.B., Clairambault, J. and Perthame, B., 2008. Analysis of a molecular structured population model with possible polynomial growth for the cell division cycle. Mathematical and Computer Modelling, 47(7), pp.699-713.

[7] Burns, F., and Tannock I., 1970. On the existence of a $G_{0}$ phase in the cell cycle. Cell Tissue Kinet, Vol.3, pp.321-334.

[8] Cooper, G. M., and Hausman, R. E., 2015. The Cell: A Molecular Approach. Published by Sinauer Associates, Inc., Seventh Edition, pp.832.

[9] Djema, W., Understanding Cell Dynamics in Cancer from Control and Mathematical Biology Standpoints: Particular Insights into the Modeling and Analysis Aspects in Hematopoietic Systems and Leukemia. 2017. Ph.D. Thesis of University Paris-Saclay (Paris-Sud), Inria Saclay, CentraleSupélec, L2S (CNRS).

[10] Djema, W., Mazenc, F. and Bonnet, C., 2017. Stability analysis and robustness results for a nonlinear system with distributed delays describing hematopoiesis. Systems \& Control Letters, 102, pp.93-101.

[11] Djema, W., Mazenc, F. and Bonnet, C., 2015, Lyapunov stability analysis of a model describing hematopoiesis. European Control Conference (ECC) IEEE, pp. 2706-2711.

[12] Djema, W., Bonnet, C., Mazenc, F., and Clairambault, J., 2018. Introducing Cell Plasticity Mechanisms into a Class of Cell Population Dynamical Systems. In the proceedings of the American Control Conference (ACC) IEEE.

[13] Djema, W., Bonnet, C., Clairambault, J., Mazenc, F., Hirsch, P., Delhommeau, F., 2017. Analysis of a model of dormancy in cancer as a state of coexistence between tumor and healthy stem cells. American Control Conference (ACC) IEEE, pp.51355140 .

[14] Elledge, S.J., 1996. Cell cycle checkpoints: preventing an identity crisis. Science, 274(5293), pp.1664-1672.

[15] Gu, K., Chen, J. and Kharitonov, V.L., 2003. Stability of timedelay systems. Berlin: Springer.

[16] Hartwell, L.H. and Kastan, M.B., 1994. Cell cycle control and cancer. Science-AAAS-Weekly Paper Edition, 266(5192), pp.1821-1828.

[17] Khalil, H.K., 2002. Nonlinear systems, 3rd. New Jewsey, Prentice Hall.

[18] Kolmanovskii, V. and Myshkis, A., 1999. Introduction to the theory and applications of functional differential equations. Kluwer Academic Publishers, Dordrecht.

[19] Lange, C. and Calegari, F., 2010. Cdks and cyclins link G1 length and differentiation of embryonic, neural and hematopoietic stem cells. Cell cycle, 9(10), pp.1893-1900.

[20] Mackey, M.C., 1978. Unified hypothesis for the origin of aplastic anemia and periodic hematopoiesis. Blood, 51(5), p.941.

[21] Malisoff, M. and Mazenc, F., 2009. Constructions of strict Lyapunov functions. Serie 320: Communications and Control Engineering. Spinger-Verlag London Ltd, U.K.

[22] Morgan, D., 2006. The Cell Cycle: Principles of Control. Primers in Biology Series, Oxford University Press, pp.297.

[23] Özbay, H., Bonnet, C., Benjelloun, H. and Clairambault, J., 2012. Stability analysis of cell dynamics in leukemia. Mathematical Modelling of Natural Phenomena, 7(1), pp.203-234.

[24] Rader, J., et al., 2013. Dual CDK4/CDK6 inhibition induces cell-cycle arrest and senescence in neuroblastoma. Clinical cancer research, 19(22), pp.6173-6182.

[25] Salomoni, P. and Calegari, F., 2010. Cell cycle control of mammalian neural stem cells: putting a speed limit on G1. Trends in cell biology, 20(5), pp.233-243.

[26] Solomon, O. and Fridman, E., 2013. New stability conditions for systems with distributed delays. Automatica, 49(11), pp.3467-3475.

[27] Tuch, B., 2006. Stem cells-a clinical update. Australian family physician, 35(9), p.719. 